\title{
Moko Disease-Causing Strains of Ralstonia solanacearum from Brazil Extend Known Diversity in Paraphyletic Phylotype II
}

\author{
Greecy M. R. Albuquerque, Liliana A. Santos, Kátia C. S. Felix, Christtianno L. Rollemberg, Adriano M. F. Silva, \\ Elineide B. Souza, Gilles Cellier, Philippe Prior, and Rosa L. R. Mariano
}

First, second, third, fourth, fifth, and ninth authors: Department of Agronomy, and sixth author: Department of Biology, Federal Rural University of Pernambuco, Av. Dom Manoel de Medeiros, s/n, Dois Irmãos, CEP 52171-900, Recife-PE, Brazil; seventh author: Anses, Plant Health Laboratory, F-97410, Saint-Pierre, La Réunion, France; and eighth author: CIRAD-INRA, UMRC53 PVBMT, F-97410, Saint-Pierre, La Réunion, France. Accepted for publication 6 May 2014.

\begin{abstract}
Albuquerque, G. M. R., Santos, L. A., Felix, K. C. S., Rollemberg, C. L., Silva, A. M. F., Souza, E. B., Cellier, G., Prior, P., and Mariano, R. L. R. 2014. Moko disease-causing strains of Ralstonia solanacearum from Brazil extend known diversity in paraphyletic phylotype II. Phytopathology 104:1175-1182.

The epidemic situation of Moko disease-causing strains in Latin America and Brazil is unclear. Thirty-seven Ralstonia solanacearum strains from Brazil that cause the Moko disease on banana and heliconia plants were sampled and phylogenetically typed using the endoglucanase (egl) and DNA repair (mutS) genes according to the phylotype and sequevar classification. All of the strains belonged to phylotype II and a

IIA-6 and IIA-24. Nevertheless, two unsuspected sequevars also harbored the Moko disease-causing strains IIA-41 and IIB-25, and a new sequevar was described and named IIA-53. All of the strains were pathogenic to banana and some of the strains of sequevars IIA-6, IIA-24, and IIA-41 were also pathogenic to tomato. The Moko disease-causing strains from sequevar IIB-25 were pathogenic to potato but not to tomato. These results highlight the high diversity of strains of Moko in Brazil, reinforce the efficiency of the egl gene to reveal relationships among these strains, and contribute to a better understanding of the diversity of paraphyletic Moko disease-causing strains of the $R$. solanacearum species complex, where the following seven distinct genetic clusters have been described: IIA-6, IIA-24, IIA-41, IIA-53, IIB-3, IIB-4, and IIB-25.
\end{abstract} portion of the strains was typed as the Moko disease-related sequevars
Different Ralstonia solanacearum strains can infect triploid banana, heliconia (Heliconia sp.), and other ornamental Musaceae plants (11) and cause Moko disease. These strains are historically known as $R$. solanacearum race 2 , biovar 1 , which is a quarantine pest (EPPO A2 list) found in Brazil that only occurs in the Amazonas, Pará, Rondônia, Roraima, Pernambuco, and Sergipe states (18). $R$. solanacearum encompasses thousands of different strains with a broad host range and unusual genetic diversity, including the two closely related strains $R$. syzygii (the causal agent of the Sumatra disease in the clove) and banana blood disease bacterium (3). Such complexity justifies the use of the term "species complex" for $R$. solanacearum $(3,4)$. However, the last two species have not been reported in Brazil. Recent studies suggest that the $R$. solanacearum species complex originated in Oceania or Indonesia, migrated to Africa, and subsequently migrated to South America and Asia, possibly before the fragmentation of the ancestral continent Gondwana $(23,31)$.

Because of this unusually large strain diversity, $R$. solanacearum was historically classified according to phenotypic and genetic properties as follow: five races based on the host range (11) and six biovars based on biochemical characteristics $(12,19)$. However, race and biovar classifications cannot mirror the broad genetic diversity of the different strains that compose the $R$. solanacearum species complex based on the development of "omics" technologies over the past decades. This issue was addressed in the 2000s and resulted in a hierarchical classification scheme by Fegan and Prior (3). This classification consisted of

Corresponding author: R. L. R. Mariano; E-mail address: rrmbac@gmail.com

http://dx.doi.org/10.1094/PHYTO-12-13-0334-R

(c) 2014 The American Phytopathological Society four phylotypes that were based on the geographical origins of the strains and 52 sequevars, which are groups of strains with highly conserved regions in a partial endoglucanase $(\mathrm{egl})$ nucleotidic sequence (26) and clones. The phylotypes were correlated with their geographical origin: phylotype I included strains from Asia, phylotype II included strains from America, phylotype III included strains from Africa and the Indian Ocean, and phylotype IV included strains from Indonesia, Japan, and Australia (22).

The classifications as phylotypes and sequevars are working definitions (22) that are presently accepted by the scientific community as best reflecting the genetic diversity of the species complex. The phylotypes can be determined from a phylogenetic analysis of the $e g l$ gene or DNA repair $(m u t S)$ gene and routinely by multiplex polymerase chain reaction (PCR) with Nmult series oligonucleotides (internal transcribed spacer region of the $16 \mathrm{~S}$ $23 \mathrm{~S}$ ribosomal RNA), which was proposed by Fegan and Prior (3). All of the Moko disease-causing strains are phylogenetically distributed within phylotype II (American origin) and further subdivided into lineages IIA and IIB $(1,4)$. More specifically, the literature has indicated the paraphyletic nature of the Moko pathogen that is distributed into four distinct sequevars: IIA-6, IIA-24, IIB-3, and IIB-4 $(1,3,4)$. The sequevars are also a working definition and subdivision of the phylotypes and they are based on a $<1 \%$ divergence of the nucleotidic sequence of the egl gene $(3,21)$. The routine detection of the Moko ecotype can be achieved by multiplex (Mmx)-PCR, which discriminates every sequevar of the Moko disease strains: IIA-6, IIA-24, IIB-3, and IIB-4 (3).

In Brazil, most of the $R$. solanacearum diversity studies were conducted on race $1(2,25,27,29)$. Unfortunately, the strains that are assigned to the obsolete race 1 classification are distributed across all four of the phylotypes. To our knowledge and based on 
the important role of the banana industry in Brazil, it is surprising that only one study has phylogenetically typed 19 Moko diseasecausing strains by Mmx-PCR (20). Typical Moko disease symptoms in the wild include yellowing and wilting of the inner leaves caused by the infection, which initiates in the rhizomes and moves toward the pseudostem; fruit are then deformed, turn black, and shrivel up. Banana plants near maturity may show no apparent symptoms but the inner pulp can still present dry rot and the plants may die. In the Philippines, Bugtok disease shows atypical symptoms confined to the inflorescence of 'Saba' and 'Cardaba' and vascular discoloration that rarely extends into the lower part of the pseudostem. These atypical symptoms are a result of the interaction of these particular cultivars and strains of $R$. solanacearum sequevar IIB-3 (4). In the state of Sergipe, which is in northeastern Brazil, the symptoms are also atypical and differ from the typical symptoms, which are found exclusively in the Amazonia region. In Sergipe, the symptoms initiate in inflorescences and cause uneven and premature ripening of fruit that internally show dry rot. The bacteria can move toward the pseudostem and cause darkening of the vascular bundles; wilting of the banana mats rarely develop, such as in Bugtok disease, which occurs in the Philippines. This Sergipe syndrome of Moko will be investigated to determine whether it results from a particular Moko strain within the dispersed genetic clusters of Moko disease-causing strain members in the disputed phylotype II.
Diversity studies are essential for the adaptation of control strategies or development of new strategies based on resistance germplasms that have yet to be developed. Determining the genetic and phenotypic variability of the Moko disease-causing strains is of the upmost importance; however, this variability remains to be described in South America (4), especially in Brazil (20). The aim of this study is to explore the pathogenic and phylogenetic diversity of $R$. solanacearum strains that originates in Brazil where recent wilting has appeared on banana (Musa sp.) and heliconia (Heliconia sp.) plants in different production areas.

\section{MATERIALS AND METHODS}

Bacterial strains and DNA typing. In total, 37 strains were obtained from symptomatic banana (Musa sp.) and heliconia (Heliconia sp.) plants (Table 1). From this total, 7 strains were obtained from the Phytobacteria Culture Collection of the Biological Institute, São Paulo, Brazil; 27 were obtained from the National Institute of Research in Amazonia, Manaus, Brazil; and 2 were obtained from the Culture Collection of the Phytobacteriology Laboratory of the Federal Rural University of Pernambuco, Recife, Brazil; and 1 from Embrapa Tabuleiros Costeiros, Aracaju, Brazil. The standard of comparison in all of the analyses was the $R$. solanacearum type strain $\mathrm{K}^{2} 0^{\mathrm{T}}$, which is the same as ATCC11696 (IBSBF292, Phytobacteria Culture Collection of the

TABLE 1. Brazilian Ralstonia solanacearum strains used in this study

\begin{tabular}{|c|c|c|c|c|c|c|}
\hline \multirow[b]{2}{*}{ Strain $^{\mathrm{a}}$} & \multirow[b]{2}{*}{ Host } & \multirow[b]{2}{*}{ Origin $^{b}$} & \multirow[b]{2}{*}{ Phyl-seq $(e g l \text { gene })^{\mathrm{c}}$} & \multirow[b]{2}{*}{ Pathogenicity (banana/tomato) } & \multicolumn{2}{|c|}{$\mathrm{GenBank}^{\mathrm{d}}$} \\
\hline & & & & & $e g l$ & mutS \\
\hline B1 & Musa sp. & Anamã/AM & IIA-24 & $+/-$ & KF889439 & KF896791 \\
\hline B11 & Banana (AAB) & Anamã/AM & IIA-24 & $+/-$ & KF875412 & KF896783 \\
\hline B13 & Banana (AAB) & Anamã/AM & IIA-24 & $+/-$ & KF889438 & KF896793 \\
\hline B133 & Musa sp. & Manacapuru/AM & IIA-24 & $+/-$ & KF875407 & KF896789 \\
\hline B14 & Banana (AAB) & Anamã/AM & IIA-24 & $+/-$ & KF875408 & KF896788 \\
\hline B15 & Banana (AAB) & Anamã/AM & IIA-24 & $+1-$ & KF875409 & KF896787 \\
\hline B17 & Banana (AAB) & Anamã/AM & IIA-24 & $+/+$ & KF875406 & KF896792 \\
\hline B3 & Banana (AAB) & Iranduba/AM & IIA-24 & $+1-$ & KF875413 & KF896782 \\
\hline B35 & Banana (AAB) & Coari/AM & IIA-24 & $+1-$ & KF889437 & KF896794 \\
\hline B5 & Banana (AAB) & Anamã/AM & IIA-24 & $+/+$ & KF875419 & KF896784 \\
\hline B6 & Banana (AAB) & Anamã/AM & IIA-24 & $+/-$ & KF875411 & KF896785 \\
\hline B67 & Banana (ABB) & Parintins/AM & IIA-24 & $+/-$ & KF875405 & KF896796 \\
\hline B9 & Banana (AAB) & Anamã/AM & IIA-24 & $+/-$ & KF875418 & KF896790 \\
\hline IBSBF1544 & Musa sp. & Amazonas & IIA-24 & $+/-$ & KF875429 & KF896769 \\
\hline IBSBF187 & Musa sp. & Humaitá/AM & IIA-24 & $+/+$ & KF875431 & KF896768 \\
\hline IBSBF188 & Musa sp. & Humaitá/AM & IIA-24 & $+/+$ & KF875430 & KF896770 \\
\hline IBSBF2571 & Musa sp. & Tabatinga/AM & IIA-24 & $+/-$ & KF875428 & KF896803 \\
\hline IBSBF615 & Musa sp. & $\mathrm{PA}$ & IIA-24 & $+/+$ & KF875417 & KF896771 \\
\hline B 105 & Banana (AAB) & Alto Solimões/AM & IIA-41 & $+/+$ & KF875410 & KF896786 \\
\hline B54 & Banana (AAB) & Manacapuru/AM & IIA-41 & $+/+$ & KF875424 & KF896795 \\
\hline B64 & Banana (AAB) & Parintins/AM & IIA-41 & $+/-$ & KF875422 & KF896776 \\
\hline B66 & Banana (AAA) & Parintins/AM & IIA-41 & $+/+$ & KF875403 & KF896775 \\
\hline B73 & Banana (AAB) & Rio Preto da Eva/AM & IIA-41 & $+/+$ & KF875423 & KF896774 \\
\hline B74 & Plantain & Tefé/AM & IIA-41 & $+/+$ & KF875404 & KF896773 \\
\hline B75 & Plantain & Tefé/AM & IIA-41 & $+/-$ & KF875421 & KF896777 \\
\hline B95 & Musa sp. & Alto Solimões/AM & IIA-41 & $+/+$ & KF875402 & KF896780 \\
\hline B96 & Musa sp. & Alto Solimões/AM & IIA-41 & $+/-$ & KF875415 & KF896778 \\
\hline B106 & Banana (AAB) & Alto Solimões/AM & IIA-41 & $+/-$ & KF875420 & KF896779 \\
\hline BV136 & Musa sp. & Paraná do Supia/AM & IIA-41 & $+/-$ & KF875414 & KF896781 \\
\hline Cotpin2 & Musa sp. & Propriá/SE & IIA-53 & $+/-$ & KF875416 & KF896797 \\
\hline $\mathrm{F} 2$ & Banana (Musa sp.) & Propriá/SE & IIA-53 & $+/-$ & KF875426 & KF896801 \\
\hline F3 & Banana (Musa sp.) & Propriá/SE & IIA-53 & $+/-$ & KF875425 & KF896802 \\
\hline IBSBF2572 & Musa sp. & Japoatã/SE & IIA-53 & $+/-$ & KF875427 & KF896772 \\
\hline IBSBF2661 & Heliconia & Abreu e Lima/PE & IIA-6 & $+/+$ & KF875432 & NA \\
\hline B4 & Banana (AAB) & Anamã/AM & IIB-25 & $+/-$ & KF889435 & KF896800 \\
\hline B7 & Banana (AAB) & Anamã/AM & IIB-25 & $+/-$ & KF889436 & KF896799 \\
\hline B10 & Banana (AAB) & Anamã/AM & IIB-25 & $+/-$ & KF889434 & KF896798 \\
\hline
\end{tabular}

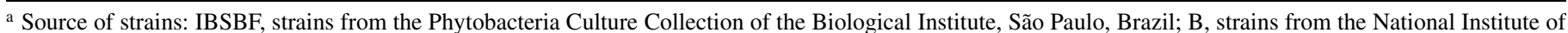
Research in Amazonia, Manaus, Brazil; F, strains from the Culture Collection of Phytobacteriology Laboratory of the Federal Rural University of Pernambuco, Recife, Brazil; Cotpin 2, strain from the Embrapa Tabuleiros Costeiros, Aracaju, Brazil.

${ }^{\mathrm{b}} \mathrm{AM}=$ Amazonas State; PA = Pará State; $\mathrm{SE}=$ Sergipe State; PE = Pernambuco State.

c Phylotype-sequevar.

$\mathrm{d} \mathrm{NA}=$ sequence not available. 
Biological Institute); the standard was isolated from tomato plants and typed as phylotype IIA sequevar 7 (IIA-7) (Table 2).

The DNA extraction of strains for the phylotype analysis was performed from the bacterial cultures grown in Kelman medium
(14) at $29^{\circ} \mathrm{C}$ for $48 \mathrm{~h}$. The bacterial genomic DNA extraction was performed with the AxyPrep Bacterial Genomic DNA MiniPrep Kit (Axygen Biosciences, Union City) according to the manufacturer's recommendations. Strain typing was performed by a

TABLE 2. GenBank reference strains of Ralstonia solanacearum complex used for phylogenetic analysis ${ }^{\mathrm{a}}$

\begin{tabular}{|c|c|c|c|c|c|c|}
\hline \multirow[b]{2}{*}{ Strain } & \multirow[b]{2}{*}{ Host } & \multirow[b]{2}{*}{ Origin } & \multirow[b]{2}{*}{ Alternative name } & \multirow[b]{2}{*}{ Phyl-seq $(e g l \text { gene })^{\mathrm{b}}$} & \multicolumn{2}{|c|}{ GenBank } \\
\hline & & & & & $e g l$ & mutS \\
\hline A3909 & Heliconia & Hawaii & RUN9 & IIA-6 & EF371812 & AY756753 \\
\hline ACH732 & Tomato & Australia & UW433 & IV-11 & GQ907150 & AY756743 \\
\hline ACH92 & Ginger & Australia & $\ldots$ & $\mathrm{I}-16$ & AF295254 & AY756764 \\
\hline CFBP2957 & Tomato & Martinique & RUN27 & IIA-36 & AF295265 & EF371845 \\
\hline CFBP2958 & Tomato & Guadeloupe (FWI) & RUN28 & IIA-39 & AF295266 & AY756806 \\
\hline CFBP2972 & Potato & Martinique (FWI) & RUN30 & IIA-35 & AF295264 & AY756807 \\
\hline CFBP3059 & Eggplant & Burkina Faso & JCG.AU28 & III-23 & AF295270 & AY756766 \\
\hline CFBP6779 & Canna Russian Red & Martinique (FWI) & ANT174-1, RUN288 & IIA-38 & EF371835.1 & EF371872 \\
\hline CFBP6783 & Heliconia & Martinique & RUN17 & IIB-4NPB & EF371852 & EF371852 \\
\hline CFBP7014 & Anthurium & Trinidad & RUN297 & IIB-51 & AF371831 & EF371875 \\
\hline CFBP7032 & Tomato & Cameroon & CMR39, RUN150 & IIA-41 & EF439726 & EF439803 \\
\hline CFBP7054 & Tomato & Cameroon & CMR121, RUN203 & IIA-52 & EF439725 & EF439800 \\
\hline CFBP7058 & Huckleberry & Cameroon & CMR134 & I-13 & EF439740 & EF439794 \\
\hline CIP10 & Potato & Peru & UW477, RUN110 & IIB-25 & AF295260 & AY756821 \\
\hline CIP240 & Potato & Brazil & RUN482 & IIB-26 & EF647739 & JF702714 \\
\hline CIP365 & Potato & Philippines & RUN47 & $\mathrm{I}-45$ & GQ907151 & AY756787 \\
\hline CIP418 & Peanut & Indonesia & MOH6 & IIB-3 & GU295005 & AY756809 \\
\hline CMR15 & Tomato & Cameroon & CFBP6941, RUN133 & III-29 & EF439743 & JF702729 \\
\hline CMR32 & Huckleberry & Cameroon & CFBP6942, RUN145 & III-29 & EF439749 & EF439773 \\
\hline CMR34 & Tomato & Cameroon & CFBP147 & IIB-1 & EF439750 & EF439810 \\
\hline CMR66 & Huckleberry & Cameroon & RUN166 & III-49 & EF439729 & EF439783 \\
\hline DGBBC1125 & Potato & Guinea & RUN369 & III-43 & GU295008 & NA \\
\hline DGBBC1138 & Potato & Guinea & RUN362 & III-44 & GU295009 & NA \\
\hline DGBBC1227 & Potato & Guinea & RUN364 & III-42 & GU295011 & NA \\
\hline GMI1000 & Tomato & French Guyana & JS753, RUN54 & $\mathrm{I}-18$ & AF295251 & AY756804 \\
\hline GMI8044 & Banana & Grenada & RUN585 & IIA-6 & GU295013 & JF702718 \\
\hline GMI8254 & Tomato & Indonesia & RUN597 & $\mathrm{I}-47$ & GU295014 & JF702719 \\
\hline IBSBF1900 & Banana & Brazil & RUN301 & IIA-24 & EF371839 & EF371871 \\
\hline IBSBF2001 & Tomato & Brazil & RUN981 & IIB-25 & GU295017 & NA \\
\hline ICMP7963 & Potato & Kenya & RUN55 & IIA-7 & AF295263 & AY766776 \\
\hline ISBSF1712 & Geranium & Brazil & RUN299 & IIB-27 & EF371833 & EF371869 \\
\hline IPO1609 & Potato & Netherlands & RUN1 & IIB-1 & EF371814 & EF371849 \\
\hline $\mathrm{J} 25$ & Tomato & Kenya & $\ldots$ & III-20 & AF295279 & AY756810 \\
\hline JT516 & Potato & Reunion & RUN160 & IIB-1 & AF295258 & AY756783 \\
\hline JT519 & Geranium & Reunion Is. & RUN471 & $\mathrm{I}-31$ & GU295032 & JF702713 \\
\hline JT525 & Geranium & Reunion & RUN60 & III-19 & AF295272 & AY756786 \\
\hline $\mathrm{K} 60^{\mathrm{Tc}}$ & Tomato & EUA & ATCC11696, IBSBF292 & IIA-7 & EF192970.1 & AY756799.1 \\
\hline M2 & Mulberry & China & RUN343 & $\mathrm{I}-48$ & FJ561067 & NA \\
\hline MAD17 & Pepper & Madagascar & RUN320 & $\mathrm{I}-46$ & GU295040 & NA \\
\hline MAFF301558 & Potato & Japan & RUN71 & IV-8 & DQ011558 & AY756812 \\
\hline Molk2 & Banana & Philippines & $\ldots$ & IIB-3 & EF371841 & EF371848 \\
\hline NCPPB1018 & Potato & Angola & RUN479 & III-21 & AF295271 & AY756772 \\
\hline NCPPB332 & Potato & Zimbabwe & RUN75 & III-22 & AF295276 & AY756760 \\
\hline NCPPB3987 & Potato & Brazil & RUN81 & IIB-28 & AF295261 & AY756785 \\
\hline $\mathrm{O} 3$ & Olive tree & China & $\ldots$ & $\mathrm{I}-44$ & FJ561069 & JF702706 \\
\hline P11 & Peanut & China & $\ldots$ & $\mathrm{I}-17$ & FJ561068 & JF702705 \\
\hline PSi7 & Tomato & Indonesia & RUN83 & IV-10 & EF371804 & AY756752 \\
\hline PSS175 & Perilla & Taiwan & $\ldots$ & $\mathrm{I}-32$ & KF913847 & NA \\
\hline PSS219 & Tomato & Taiwan & $\ldots$ & $\mathrm{I}-34$ & FJ561167 & JF702700 \\
\hline PSS358 & Tomato & Taiwan & $\ldots$ & $\mathrm{I}-15$ & EU407298 & JF702699 \\
\hline PSS81 & Tomato & Taiwan & $\ldots$ & I-14 & FJ561066 & JF702701 \\
\hline R229 (BDB) & Banana & Indonesia & RUN62 & IV-10 & GU295045 & AY756811 \\
\hline R24 (RSY) & Clove & Indonesia & $\ldots$ & IV-9 & JF702321 & JF702735 \\
\hline R292 & White mulberry & China & RUN91 & $\mathrm{I}-12$ & AF295255 & AY756801 \\
\hline RUN549 & Tomato & Trinidad & RF38 & IIA-37 & JF702309 & JF702716 \\
\hline T1-UY & Tomato & Uruguay & RUN448 & IIA-50 & GU295049 & JF702712 \\
\hline UQRS555 & Ginger & Mauritius & $\ldots$ & $\mathrm{I}-33$ & KF913848 & NA \\
\hline UQRS565 & Ginger & Thailand & $\ldots$ & $\mathrm{I}-30$ & KF913846 & NA \\
\hline UW163 & Plantain & Peru & RUN586 & IIB-4 & GU295052 & AY756779 \\
\hline UW170 & Heliconia & Colombia & RUN262 & IIB-4 & DQ011550 & JF702702 \\
\hline UW181 & Plantain & Venezuela & RUN454 & IIA-6 & GU295053 & AY756754 \\
\hline UW469 & Potato & Brazil & JT652 & IIA-40 & DQ657612 & AY756808 \\
\hline
\end{tabular}

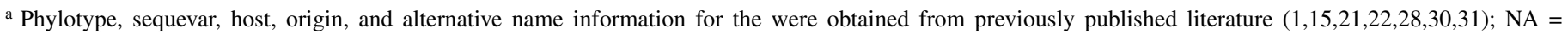
sequence not available.

b Phylotype-sequevar.

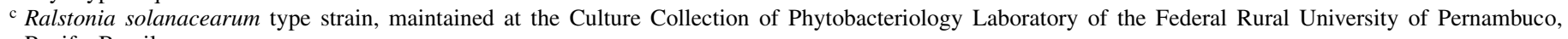
Recife, Brazil. 
phylotype-specific multiplex (Pmx)-PCR, which characterized the appurtenance to the $R$. solanacearum species complex and phylotypes (3); strains belonging to phylotype II were further characterized by the specific Mmx-PCR for the Moko diseasecausing strains $(3,28)$.

PCR amplification and partial DNA sequencing of the $e g l$ and mutS genes. The PCR amplification of a 750-bp region of the egl gene was performed using the primer pair Endo-F $\left(5^{\prime}\right.$ ATGCATGCCGCTGGTCGCCGC-3') and Endo-R (5'-GCGTTG CCCGGCACGAACACC-3') (21). The reaction mixture $(50 \mu \mathrm{l}$ total volume) contained $2 \times$ PCR Master Mix (Thermo Scientific, San Jose, CA), $0.5 \mu \mathrm{M}$ primers, and $100 \mathrm{ng}$ of DNA. The reaction mixtures were heated for $9 \mathrm{~min}$ at $96^{\circ} \mathrm{C}$ and followed by 30 cycles $\left(95^{\circ} \mathrm{C}\right.$ for $1 \mathrm{~min}, 55^{\circ} \mathrm{C}$ for $40 \mathrm{~s}$, and $72^{\circ} \mathrm{C}$ for $2 \mathrm{~min}$ ), with a final extension step for $10 \mathrm{~min}$ at $72^{\circ} \mathrm{C}$. The amplification of the 758-bp fragment of the mutS gene was performed in a total volume of $50 \mu \mathrm{l}$ that contained $2 \times$ PCR Master Mix, $2.5 \%$ dimethyl sulfoxide, $100 \mathrm{ng}$ of DNA, and $1 \mu \mathrm{M}$ primers mutSRsF1570 (5'-ACAGCGCCTTGAGCCGTACA-3') and mutSRsR1926 (5'-GCTGATCACCGGCCCGAACAT-3') (28). The reaction mixtures were heated for $5 \mathrm{~min}$ at $96^{\circ} \mathrm{C}$ and followed by 35 cycles $\left(94^{\circ} \mathrm{C}\right.$ for $1 \mathrm{~min}, 66^{\circ} \mathrm{C}$ for $1 \mathrm{~min}$, and $72^{\circ} \mathrm{C}$ for $\left.90 \mathrm{~s}\right)$, with a final extension step for $5 \mathrm{~min}$ at $72^{\circ} \mathrm{C}$. The PCR products were submitted to electrophoresis on a $1.5 \%$ agarose gel in $0.5 \times$ Tris-borate-EDTA buffer along with the GeneRuler 100-bp DNA ladder. The purification of the PCR products was performed with the PCR Clean-up Kit (Axygen Biosciences) and double strands were sequenced by Macrogen (Seoul, South Korea).

Sequence analysis and construction of phylogenetic trees. The alignment of partial sequences of the egl and mutS genes were performed with ARB Software Environment (15,17) (http:// www.arb-home.de/). The phylogenetic reconstruction of strains was achieved with PhyML software (9) using the F81 nucleotide substitution model, which allows base frequencies to vary from 0.25 and aLRT branch support statistics while using the BEST topology search (best of NNI and SPR search). The egl and mutS sequences identified in this study were deposited in the GenBank database (Table 1).

Pathogenicity tests. The Moko strains and type strain $\mathrm{K} 60^{\mathrm{T}}$ (tomato, EUA) were tested for pathogenicity on 'Williams' banana plantlets (Musa sp.; Cavendish Group, AAA) obtained by micropropagation. The plantlets were grown in 500-ml plastic pots that contained Carolina Padrão substrate (sphagnum moss cake, expanded vermiculite, dolomitic limestone, gypsum, and traces of NPK) (Carolina Soil, Vera Cruz, Brazil) until six leaves were fully developed. The plantlets were then inoculated by injecting $1 \mathrm{ml}$ of an $R$. solanacearum strain suspension $\left(10^{8}\right.$ $\mathrm{CFU} / \mathrm{ml}$ ) into the pseudostem. The plants were kept in a greenhouse with a mean temperature of $36 \pm 2{ }^{\circ} \mathrm{C}$ and mean relative humidity of $85 \%$ and checked for 40 days to observe the occurrence of Moko disease symptoms.

The ability of the Brazilian $R$. solanacearum Moko diseasecausing strains to infect the tomato plants was studied with the plantlets of 'Santa Clara', which is widely grown in Brazil and susceptible to bacterial wilt. Plants that were grown in 500-ml plastic pots that contained the Carolina Padrão substrate for 25 days were inoculated by the root wound method (5), then kept in a greenhouse with mean temperature and relative humidity values of $38 \pm 2{ }^{\circ} \mathrm{C}$ and $88 \%$, respectively, and assessed for 30 days. The strain pathogenicity was recorded using a qualitative criterion (i.e., - for symptom-free plants and + for wilting or dead plants).

According to the phylogenetic analysis of the egl gene sequence, three Moko strains (B4, B7, and B10) were assigned to sequevar IIB-25 (reference strain CIP10, potato, Peru). This sequevar is not known to host the Moko strains; therefore the strains were further investigated for their ability to cause wilt in the 'Aracy' potato (Solanum tuberosum) plant within 21 days after budding. The plants were inoculated following the same methodology as the tomato pathogenicity tests. Once symptoms appeared, the bacterial streaming tests and reisolation in triphenil tetrazolium chloride (TZC) agar medium were performed. The strain IBSBF455 (potato, Brazil) was used as the standard strain for comparison.

Based on the phylogenetic analyses, 11 Brazilian $R$. solanacearum Moko disease-causing strains were assigned to sequevar IIA-41 (reference strain CFBP7032, tomato, Cameroon) and three Brazilian strains were closely related to sequevar IIB-25. To investigate the relationship between this unsuspected phylogenetic position and pathogenicity, representative strains of sequevar IIA41 (CFPB7032, tomato, Cameroon; RUN434, irrigation water, French Guiana; RUN117, bell pepper, Brazil; and RUN118, chili, Brazil) and sequevar IIB-25 (CIP10, potato, Peru; RUN981, tomato, Brazil; and RUN1340 and RUN1341, potato, Iran) were inoculated on 'Grand Naine' banana (Cavendish group) according

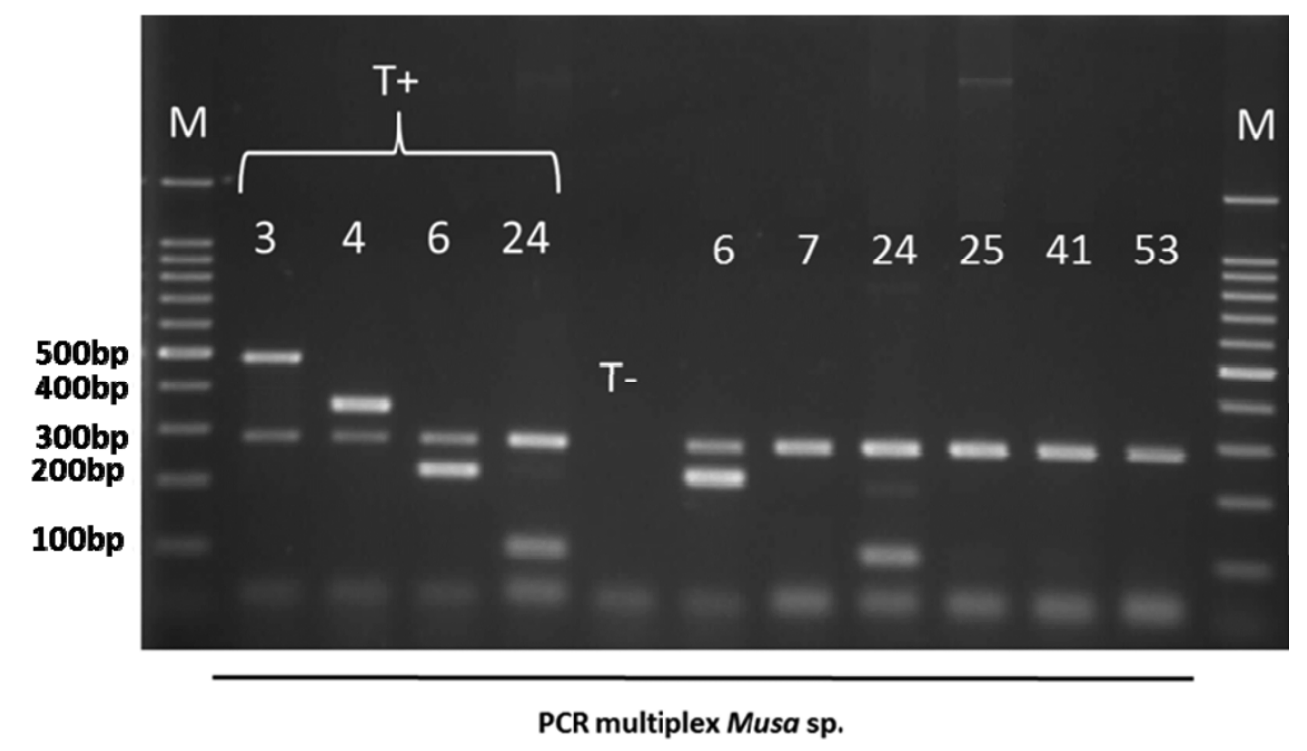

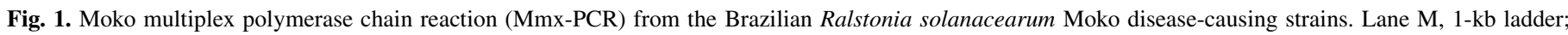

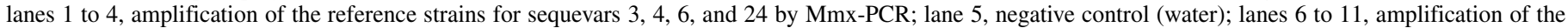

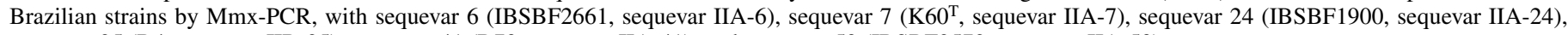
sequevar 25 (B4, sequevar IIB-25), sequevar 41 (B73, sequevar IIA-41), and sequevar 53 (IBSBF2572, sequevar IIA-53). 
to Cellier and Prior (1). The pathogenicity test was performed in a high-security quarantine facility (NS3-Rotoplan, Cirad, Réunion Island, France). Banana plants with five fully developed leaves were inoculated by injecting the pathogen into the pseudostem and depositing the inoculum manually on wounded roots. Symptom development was visually assessed every 3 days for 30 days.

\section{RESULTS}

DNA typing. The Pmx-PCRs revealed two amplicons: the 282-bp amplicon specific to the $R$. solanacearum species complex (internal marker) and the 372-bp amplicon, indicating that all of the Brazilian Moko disease-causing strains belonged to phylotype II. The Mmx-PCR identified the sequevars IIA-6 (220 bp) and IIA-24 (100 bp) by using the VC46F/VC46E and SI28F/SI28R primer pairs, respectively. Other strains only amplified the 282-bp amplicon that was specific to $R$. solanacearum $(759 / 760$ primer pair); however, a specific amplicon was not amplified for any known Moko sequevars (Fig. 1).

Phylogenetic analysis. The phylotype and sequevar strain typing was based on a phylogenetic reconstruction from a partial sequence of the $e g l$ sequences along with reference $e g l$ sequences from the GenBank database that covered the know sequevar diversity within the $R$. solanacearum species complex. Trees constructed with the $e g l$ and $m u t S$ sets of sequences were generally consistent with previously reported topological differences (22) (Figs. 2 and 3), in which phylotype II was more closely related to phylotype III in the phylogenetic egl tree and closer to phylotype I in the phylogenetic mutS tree.

Among the Brazilian strains, the phylogenetic egl tree revealed the presence of the Moko disease-related sequevars IIA-6 (IBSBF2661) and IIA-24 ( $n=18$ strains) (Fig. 2). Furthermore, three new clusters of strains did not group with any previously described Moko disease clusters. The first group, which is reported here as a new sequevar IIA-53, consisted of strains F2, F3, Cotpin2, and IBSBF2572, which were all from Sergipe State. The second group consisted of 11 strains related to strain CFBP7032 (tomato, Cameroon) and assigned to the wellestablished sequevar IIA-41. The third group included clustered strains B4, B7, and B10 and was closely related to the potato strain CIP10 (Peru) assigned to the known sequevar IIB-25 (Fig. 1). The phylogenetic mutS tree (Fig. 3) also unraveled the Moko strains in the sequevars IIA-24 ( $n=17$ strains), IIA-41 $(n=12$ strains), and IIA-53 (F2, F3, and IBSBF2571). An evaluation of

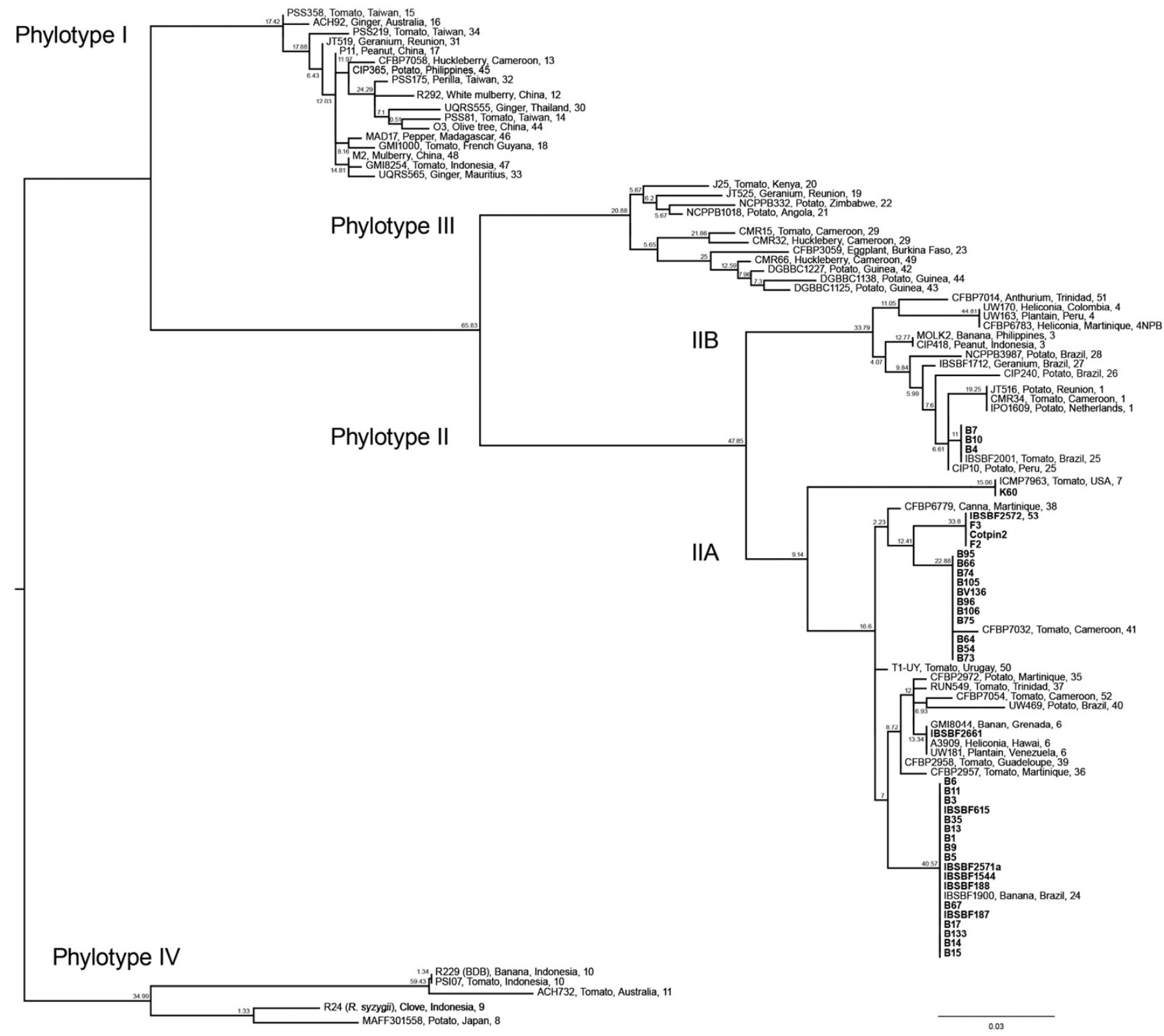

Fig. 2. Phylogenetic reconstruction based on the partial endoglucanase ( $\mathrm{egl}$ ) gene sequences from Brazilian Moko disease-causing Ralstonia solanacearum strains using the PhyML software and F81 nucleotide substitution model along with aLRT branch support statistics while using the BEST topology search (best of NNI and SPR search). 
the Moko strains B4, B7, and B10 indicated that they were incongruent in their phylogenetic assignment between the phylogenetic egl and mutS trees; in the mutS tree, they were grouped in a distinct cluster within phylotype IIB and more closely related to sequevar IIB-3 strains MOLK2 (Musa sp., Indonesia), CFBP1409 (Musa sp., Honduras), UW9 (Heliconia sp., Costa Rica), and CFBP1183 (Heliconia sp., Costa Rica) (Fig. $3)$. There was also incongruence between the egl and mutS trees for strain IBSBF2571, which was grouped in the IIA-24 sequevar in the $e g l$ tree and IIA-53 in the mutS tree.

Pathogenicity testing. All of the Brazilian strains isolated from the banana and heliconia specimens induced characteristic Moko disease symptoms in the banana plants 15 to 30 days postinoculation (dpi). The Santa Clara tomato plant also presented wilt symptoms following the inoculation of 12 of 37 strains (Table 1), including the type strain $\mathrm{K}_{60}^{\mathrm{T}}$ at 15 to $30 \mathrm{dpi}$. Among the 12 strains that were found to be pathogenic to tomato, 1 belonged to sequevar IIA-6, 5 belonged to IIA-24, and 6 belonged to IIA-41. Strains from sequevars IIB-25 $(n=3)$, IIA-53 $(n=4)$, IIA-41 $(n=5)$, and IIA-24 $(n=14)$ were not found to be pathogenic to tomato (Table 1 ).

The Moko strains B4, B7, and B10 and reference strain CIP10 (potato, Peru) were phylogenetically assigned to sequevar IIB-25 and found to be pathogenic to potato under greenhouse conditions; this result is consistent with the phenotype of the type strain IBSBF455 (potato, Brazil). The incubation period was 15 days. In all of the cases, the bacterial streaming test was positive and strains were reisolated in TZC medium. The reference strains belonging to sequevar IIA-41 obtained from other hosts (CFPB7032, RUN434, RUN117, and RUN118), IIB-25 (CIP10, RUN981, RUN1340, and RUN1341), and IIA-7 (K60 $\left.{ }^{\mathrm{T}}\right)$ were characterized as nonpathogenic to banana because the plants did not develop any symptoms 4 weeks after inoculation.

\section{DISCUSSION}

The Moko disease caused by $R$. solanacearum in banana plants is a quarantine pest in Europe (EPPO A2 list) and Brazil, where it has been observed in the northern and northeastern regions and has severely limited crop production in some areas (27). This study investigates the genetic and pathogenic diversity and phylogenetic positions of $37 R$. solanacearum Moko disease-causing strains from Brazil. It was not surprising that this relatively low sampling $(n=37)$ mirrored such a broad level of genetic diversity because limited studies have been conducted in Brazil on the genetic diversity of the $R$. solanacearum Moko disease-causing strains. Only scarce information by Pinheiro et al. (20) showed the existence of potential unsuspected genetic groups among the Moko disease agents within phylotype II.

The sequevar is a working definition based on the partial sequence of the $e g l$ gene. The phylogenetic assignments based on egl typing are congruent with the organismal phylogeny based on

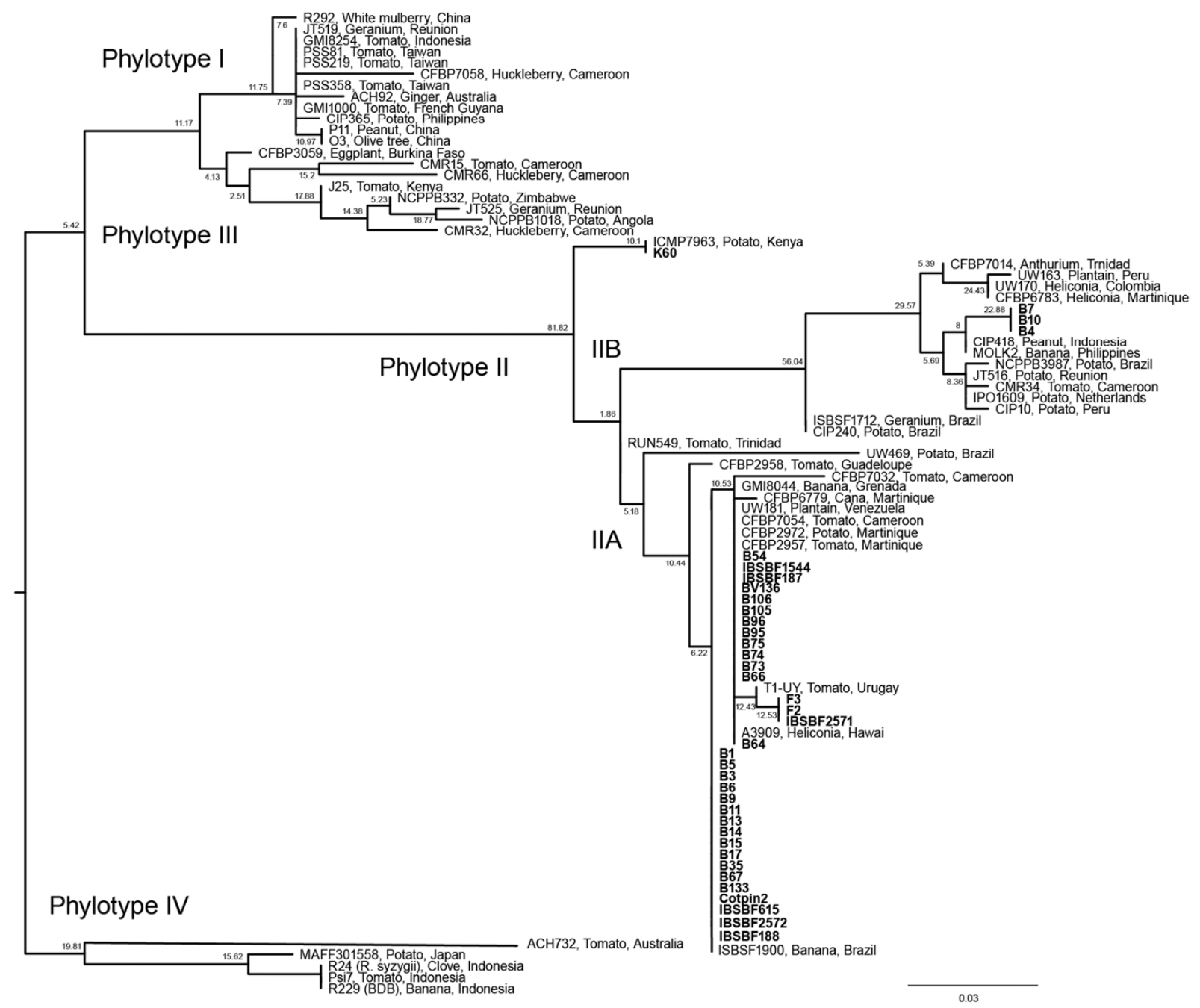

Fig. 3. Phylogenetic reconstruction based on the partial DNA repair (mutS) gene sequences from Brazilian Moko disease-causing Ralstonia solanacearum strains using the PhyML software and F81 nucleotide substitution model along with aLRT branch support statistics while using the BEST topology search (best of NNI and SPR search). 
pangenomic DNA microarray hybridization (8), and this study confirmed that the $e g l$ gene is a reliable tool for determining the phylogenetic relationships of a particular strain within the $R$. solanacearum species complex $(3,4,28)$.

All of the $R$. solanacearum Moko strains are historically known to infect species of both Musaceae and Heliconiaceae but only some of them infect members of the Solanaceae family under greenhouse conditions (1). Based on the hierarchical classification proposed by Fegan and Prior (4), these strains are typed into four sequevars in the phylotype II: IIA-6, IIA-24, IIB-3, and IIB-4. In this study, some of the Brazilian Moko disease-causing strains belonged to sequevars IIA-6 and IIA-24, whereas other strains were surprisingly assigned to sequevars IIA-41 and IIB25. The Moko strains B4, B7, and B10 in sequevar IIB-25 were characterized as pathogenic to banana and potato and phylogenetically related to strain CIP10 (potato, Peru) in the phylogenetic egl tree; however, they showed a distinct phylogenetic cluster in the phylogenetic mutS tree. Such an unexpected assignment of Moko and non-Moko strains sharing the same phylogenetic position was also observed by Wicker et al. (30) in Martinique. This work reported on the emerging strains within the Moko sequevar IIB-4 that were shown to be new pathological variants. These emerging strains were nonpathogenic to banana (IIB4-NPB) but highly aggressive to eggplant, pepper, and tomato, and they even broke down the resistance in tomato 'Hawaii 7996' (30). A new emergent strain of $R$. solanacearum (RS37) obtained from geranium (Pelargonium sp.) in Florida colonized triploid banana and reduced the growth of the plant without causing wilting. Strain RS37 was phylogenetically related (egl gene) to the Martinique strain CFBP6801 (from Heliconia caribaea) and belonged to phylotype II; however, its sequevar was not defined (13). The phylogenetic position of sequevar IIB25 was framed by the banana Moko IIB-3 strains and potato brown-rot IIB-1 strains; therefore, it may constitute an evolutionary link between the two well-known ecotypes and highlight their potential importance in evolutionary studies for understanding host adaptation.

Furthermore, the Moko disease-causing strains F2, F3, Cotpin2, and IBSBF2572 did not cluster with any previously referenced sequevars but formed the new sequevar IIA-53. The strains IBSBF2572 and Cotpin2 clustered with the strain-typed sequevar IIA-24 in the mutS phylogenetic tree; however, the sequevars were based on the egl phylogenetic tree and these two strains will remain in sequevar IIA-53 until further investigation. Therefore, it was confirmed that the $m u t S$-tree resolution was insufficient to support the sequevar typing of the Moko strains. The new sequevar IIA-53 extended the known phylogenetic diversity of the paraphyletic Moko strains to seven different sequevars that are distributed as phylotypes IIA (IIA-6, IIA-24, IIA-41, and IIA-53) and IIB (IIB-3, IIB-4, and IIB-25). In addition to the newly proposed sequevar IIA-53, the existence of an even larger number of unreported sequevars in Brazil and South America remains possible. This idea is supported by the fact that the last Mokodisease-related sequevar was detected in Brazil (sequevar IIA-24) (4). Brazilian strains IIA-6 and IIA-24 amplified the expected amplicons in the specific Mmx-PCR; however, the Moko strains from sequevars IIA-41, IIA-53, and IIB-25 only amplified the internal marker of the $R$. solanacearum species, which indicated the necessity to develop additional molecular tools to produce reliable diagnostic protocols for all of the Moko sequevars.

All of the Brazilian $R$. solanacearum Moko disease-causing strains tested in this study were pathogenic to banana plants and showed characteristic symptoms of the disease, such as browning of the xylem vessels and wilting until death. These strains displayed variable pathogenic levels on the tomato plants, and no correlation with the sequevar classification was observed among strain IBSBF2661 (IIA-6) and 11 others pathogenic strains, which is consistent with Cellier and Prior (1), who reported that some strains are pathogenic to tomato plants. To our knowledge, the Moko disease-causing strains have never been witnessed in fieldgrown infected tomato or potato plants, although these strains could be virulent on susceptible potato and tomato plants. This raises a difficult question regarding the role of host adaptation as a bottleneck evolutionary process and its link with the development of strains that are likely to cause epidemics, such as Moko (1).

The correlation between the new syndrome in the state of Sergipe, known here as the "Sergipe facies", and new sequevar IIA-53 confirmed our hypothesis that the syndrome is caused by particular Moko strains in phylotype II. The group reported here as sequevar IIA-53 included strains from the state of Sergipe in northeastern Brazil that were obtained from the peduncle of banana plants showing atypical symptoms, which were similar to the Bugtok disease in Philippines and different from the typical symptoms found in the northern region of Brazil. These strains caused infection that was initiated in the inflorescence and followed by uneven and premature ripening of fruit that internally showed dry rot, and the disease occurred under an aggregated spatial pattern. The bacteria can move toward the pseudostem and cause darkening of the vascular system but it will never cause wilting or yellowing of the plants.

Sequevar IIB-25 included strains from Amazonas State that were found to be pathogenic to potato under experimental conditions. This ability to wilt members of the families Musaceae, Heliconiaceae, and Solanaceae from the Moko ecotype is important and should be considered from both an epidemiological and evolutionary viewpoint.

Central America and northern South America are reported as the centers of origin $(10,24)$ and diversification of the Moko disease-causing strains, which might be related to the large geographical size of the banana production area. Because the phylogenetic analysis of the egl gene represents an organismal phylogeny, we can predict three possible hypotheses to explain this paraphyly: (i) the Moko disease-causing ability developed from closely related but genetically different strains (convergent evolution), (ii) all of the clusters in the phylogeny of $R$. solanacearum species complex except the Moko strains lost the ability to cause the disease in banana, and (iii) horizontal gene transfer (HGT) between pathogenic and nonpathogenic strains in the banana occurred and resulted in new pathotypes (4). Relative to this last hypothesis, HGT is known as a major driving force in bacterial evolution (6). By analyzing the comparative genomic hybridization on microarrays, Guidot et al. (7) demonstrated that DNA blocks of $30 \mathrm{~kb}$ and 33 genes could be integrated during a single transformation event. Lefeuvre et al. (16) reported that most of the putative HGT events were detected between strains clustered with the Molk2 strain (IIB-3) and in recombinogenic strains of phylotypes I and IIA. Moreover, a limited number of HGT events was found among strains of phylotypes I, III, and IV. From the multilocus sequence analysis data, Wicker et al. (31) reported that the recombination between strains, particularly within phylotype IIB, had a different shift pattern, with phylotype IIB being nearly clonal and phylotype IIA being recombinogenic, highly diverse, and expanding.

This study highlights that, in Brazil, Moko disease is caused by $R$. solanacearum strains from a broad genetic diversity. In addition to the well-characterized Moko sequevars, two sequevars (IIA-41 and IIB-25) previously unrelated to Moko disease were typed as pathogenic to banana, with similar symptoms. Additional environmental strains must be investigated to determine if the Sergipe facies of the Moko disease in the field can be attributed to the new sequevar IIA-53 only. This new phylogenetic aspect of the Moko ecotype must be further investigated and characterized to provide (i) clues for epidemiological studies, (ii) guidance for disease control programs, and (iii) new molecular diagnostic tools for Moko disease-causing strains. 


\section{ACKNOWLEDGMENTS}

We thank the National Council for Scientific and Technological Development $(\mathrm{CNPq})$ for the scholarship to $\mathrm{G}$. $\mathrm{M}$. R. Albuquerque and research fellowships to A. M. F. Silva (Proc. 101000/2011-1), R. L. R. Mariano (Proc. 309697/2011-5), and E. B. Souza. Financial support was also provided by the CNPq/PNPD (Proc. 560.606/2010-9). We thank R. Coelho Neto from the National Institute of Research in Amazonia, Manaus, Brazil and V. Talamini from the Embrapa Tabuleiros Costeiros, Aracaju, Brazil for donating most of the strains used in this study; and S. Arriba and J.-J. Chéron from the CIRAD, UMR Peuplement Végétaux et Bioagresseurs en Milieu Tropical for their technical assistance.

\section{LITERATURE CITED}

1. Cellier, G., and Prior, P. 2010. Deciphering phenotypic diversity of Ralstonia solanacearum strains pathogenic to potato. Phytopathology 100:1250-1261.

2. Costa, S. B., Ferreira, M. A. S. V., and Lopes, C. A. 2007. Diversidade patogênica e molecular de Ralstonia solanacearum da Região Amazônica brasileira. Fitopatol. Bras. 32:285-294.

3. Fegan, M., and Prior, P. 2005. How complex is the Ralstonia solanacearum species complex? Pages 449-461 in: Bacterial Wilt Disease and the Ralstonia solanacearum Species Complex. C. Allen, P. Prior, and A. C. Hayward, eds. American Phytopathological Society, St. Paul, MN.

4. Fegan, M., and Prior, P. 2006. Diverse members of the Ralstonia solanacearum species complex cause bacterial wilts of banana. Australas. Plant Pathol. 35:93-101.

5. Felix, K. C. S., Souza, E. B., Michereff, S. J., and Mariano, R. L. R. 2012. Survival of Ralstonia solanacearum in infected tissues of Capsicum aпnиит and in soils of the state of Pernambuco, Brazil. Phytoparasitica 40:53-62.

6. Gogarten, J. P., and Townsend, J. P. 2005. Horizontal gene transfer, genome innovation and evolution. Nat. Rev. Microbiol. 3:679-687.

7. Guidot, A., Coupat, B., Fall, S., Prior, P., and Bertolla, F. 2009. Horizontal gene transfer between Ralstonia solanacearum strains detected by comparative genomic hybridization on microarrays. ISME J. 3:549-562.

8. Guidot, A., Prior, P., Schoenfeld, J., Carrère, S., Genin, S., and Boucher, C. 2007. Genomic structure and phylogeny of the plant pathogen Ralstonia solanacearum inferred from gene distribution analysis. J. Bacteriol. 189:377-387.

9. Guindon, S., Dufayard, J. F., Lefort, V., Anisimova, M., Hordijk, W., and Gascuel, O. 2010. New algorithms and methods to estimate maximumlikelihood phylogenies: Assessing the performance of PhyML 3.0. Syst. Biol. 59:307-321.

10. Hayward, A. C. 1991. Biology and epidemiology of bacterial wilt caused by Pseudomonas solanacearum. Annu. Rev. Phytopathol. 29:65-87.

11. Hayward, A. C. 1994. The hosts of Pseudomonas solanacearum. Pages 924 in: Bacterial Wilt: The Disease and its Causative Agent, Pseudomonas solanacearum. A. C. Hayward and G. L. Hartman, eds. CAB International, Wallingford, UK.

12. He, L. Y., Sequeira, L., and Kelman, A. 1983. Characteristics of strains of Pseudomonas solanacearum. Plant Dis. 67:1357-1361.

13. Hong, J. C., Norman, D. J., Reed, D. L., Momol, M. T., and Jones, J. B. 2012. Diversity among Ralstonia solanacearum strains isolated from the southeastern United States. Phytopathology 102:924-936.

14. Kelman, A. 1954. The relationship of pathogenicity in Pseudomonas solanacearum to colony appearance on a tetrazolium medium. Phytopathology 44:693-695.

15. Lebeau, A., Daunay, M. C., Frary, A., Palloix, A., Wang, J. F., Dintinger, J., Chiroleu, F., Wicker, E., and Prior, P. 2011. Bacterial wilt resistance in tomato, eggplant and pepper: Genetic resources challenged with the multifaceted Ralstonia solanacearum species complex. Phytopathology 101:154-165.

16. Lefeuvre, P., Cellier, G., Remenant, B., Chiroleu, F., and Prior, P. 2013. Constraints on genome dynamics revealed from gene distribution among the Ralstonia solanacearum species. PLoS One 8:e63155.

17. Ludwig, W., Strunk, O., Westam, R., Richter, L., Meier, H., Yadhukumar, Buchner, A., Lai, T., Steppi, S., Jobb, G., Förster, W., Brettske, I., Gerber, S., Ginhart, A. W., Gross, O., Grumann, S., Hermann, S., Jost, R., König, A., Liss, T., Lüßmann, R., May, M., Nonhoff, B., Reichel, B., Strehlow, R., Stamatakis, A., Stuckmann, N., Vilbig, A., Lenke, M., Ludwig, T., Bode, A., and Schleifer, K.-H. 2004. ARB: A software environment for sequence data. Nucleic Acids Res. 32:1363-1371.

18. MAPA. 2007. Ministério da Agricultura, Pecuária e Abastecimento. Brasil. Instrução Normativa No. 52 de 20/11/2007. http://extranet.agricultura.gov.br/ sislegis-consulta/consultarLegislacao.do?operacao=visualizar\&id $=18212$

19. Meng, F. 2013. Ralstonia solanacearum species complex and bacterial wilt disease. J. Bacteriol. Parasitol. 4:e119.

20. Pinheiro, C. R., Amorim, J. A. E., Diniz, L. E. C., Silva, A. M. F., Talamini, V., and Souza-Jr., M. T. 2011. Diversidade genética de isolados de Ralstonia solanacearum e caracterização molecular quanto à filotipos e sequevares. Pesq. Agropec. Bras. 46:593-602.

21. Poussier, S., Prior, P., Luisetti, J., Hayward, C., and Fegan, M. 2000. Partial sequencing of the $h r p B$ and endoglucanase genes confirms and expands the known diversity within the Ralstonia solanacearum species complex. Syst. Appl. Microbiol. 23:479-486.

22. Prior, P., and Fegan, M. 2005. Recent developments in the phylogeny and classification of Ralstonia solanacearum. Pages 127-136 in: Proc. First Int. Symp. Tomato Dis. M. T. Momol, P. Ji, and J. B. Jones, eds. Acta Horticulturae, Orlando, FL.

23. Remenant, B., Coupat-Goutaland, B., Guidot, A., Cellier, G., Wicker, E., Allen, C., Fegan, M., Pruvost, O., Elbaz, M., Calteau, A., Salvignol, G., Mornico, D., Mangenot, S., Barbe, V., Médigue, C., and Prior, P. 2010. Genomes of three tomato pathogens within the Ralstonia solanacearum species complex reveal significant evolutionary divergence. BMC Genomics 11:379.

24. Sequeira, L., and Averre, C. 1961. Distribution and pathogenicity of strains of Pseudomonas solanacearum from virgin soils in Costa Rica. Plant Dis. 45:435-440.

25. Silveira, J. R. P., Duarte, V., Moraes, M. G., Oliveira, A. M. R., Barni, V., and Maciel, J. L. N. 2005. Caracterização de estirpes de Ralstonia solanacearum isoladas de plantas de batata com murcha bacteriana por PCR-rep e RAPD. Fitopatol. Bras. 30:615-622.

26. Siri, M. I., Sanabria, A., and Pianzzola, M. J. 2011. Genetic diversity and aggressiveness of Ralstonia solanacearum strains causing bacterial wilt of potato in Uruguay. Plant Dis. 95:1292-1301.

27. Talamini, V., Silva, A. M. F., Almeida, M. A., Moraes, A. C., Warwick, D. R. N., Nascimento, M. P. A., and Devi, C. K. 2010. Situação do Moko da bananeira no estado de Sergipe. http://www.cpatc.embrapa.br/ publicacoes_2010/doc_159.pdf

28. Toukam, G. M. S., Cellier, G., Wicker, E., Guilbaud, C., Kahane, R., Allen, C., and Prior, P. 2009. Broad diversity of stains in Cameroon. Plant Dis. 93:1123-1130.

29. Viana, F. C., Berger, I. J., and Duarte, V. 2012. Caracterização de populações de Ralstonia solanacearum Smith em tabaco (Nicotiana tabacum L.) no Brasil. Trop. Plant Pathol. 37:123-129.

30. Wicker, E., Grassart, L., Coranson-Beaudu, R., Mian, D., Guilbaud, C., Fegan, M., and Prior, P. 2007. Ralstonia solanacearum strains from Martinique (French West Indies) exhibiting a new pathogenic potential. Appl. Environ. Microbiol. 73:6790-6801.

31. Wicker, E., Lefeuvre, P., de Cambiaire, J. C., Poussier, S., and Prior, P. 2012. Contrasting recombination patterns and demographic histories of the plant pathogen Ralstonia solanacearum inferred from MLSA. ISME J. 6:961-974. 